\title{
Epidemiological distribution of primary central nervous system tumors in the Western Province of Saudi Arabia: a local registry from neuroscience-affiliated centers
}

\author{
Maher Kurdi ${ }^{1}$, Nadeem Shafique Butt ${ }^{2}$, Saleh Baeesa ${ }^{3}$, Badrah Alghamdi ${ }^{4}$, Yazid Maghrabi ${ }^{5}$, \\ Anas Bardeesi ${ }^{5}$, Rothaina Saeedi ${ }^{3}$, Ahmed I. Lary ${ }^{6}$ \\ 'Department of Pathology, Faculty of Medicine, King Abdulaziz University, Rabigh, Kingdom of Saudi Arabia; ${ }^{2}$ Department of Family and \\ Community Medicine, Faculty of Medicine, King Abdulaziz University, Jeddah, Kingdom of Saudi Arabia; ${ }^{3}$ Division of Neurosurgery, Department \\ of Surgery, Faculty of Medicine, King Abdulaziz University, Jeddah, Kingdom of Saudi Arabia; ${ }^{4}$ Department of Physiology, Faculty of Medicine, \\ King Abdulaziz University, Jeddah, Kingdom of Saudi Arabia; ${ }^{5}$ Department of Neuroscience, King Faisal Specialist Hospital and Research Center, \\ Jeddah, Kingdom of Saudi Arabia; ${ }^{6}$ Section of Neurosurgery, Department of Surgery, King Abdulaziz Medical City, Jeddah, Kingdom of Saudi \\ Arabia
}

OBJECTIVES: Central nervous system (CNS) tumors are a major and growing global healthcare challenge. Western Saudi Arabia has an inconsistent data registry; therefore, the epidemiology of CNS tumors is unclear across the country. This study is aimed to assemble the epidemiological matrix of CNS tumors in the Western Province of Saudi Arabia.

METHODS: A retrospective analysis was performed using clinical data obtained from 3 neuroscience centers in Western Saudi Arabia in the period 2014-2019. The sample size included 663 adult and pediatric cases from the local and expatriate populations diagnosed with CNS tumors. The distributions of age, sex, clinical presentation, tumor location, type of surgery, histological subtype, genetic characteristics, and recurrence rate were explored.

RESULTS: The analysis included 500 adult cases and 163 pediatric cases up to 18 years of age with a male-to-female ratio of 1.16. The mean age at diagnosis was $38.0 \pm 22.6$ years. The supratentorium was the most common location $(n=515,77.7 \%)$. Most patients presented with headache $(n=298,44.9 \%)$, followed by a focal neurological deficit $(19.9 \%)$. The most common primary CNS tumor was glioblastoma $(n=234,35.3 \%)$, followed by meningioma $(n=100,15.1 \%)$. The recurrence rate after surgery was estimated to be $40.9 \%$ among all CNS tumors.

CONCLUSIONS: This is the first tumor registry of Western Province of Saudi Arabia that describes the distribution of primary CNS tumors and highlights their epidemiological matrix. Several incidence trends in terms of histological type, age group, sex, location, and recurrence were determined, and some genetic characteristics were recognized.

KEY WORDS: Central nervous system tumour, Brain tumour, Epidemiology, Incidence, Saudi Arabia, Asia

\section{Correspondence: Maher Kurdi}

Department of Pathology, Faculty of Medicine, King Abdulaziz University, Rabigh 21589, Kingdom of Saudi Arabia

E-mail: Ahkurdi@kau.edu.sa

Received: Mar 18, 2021 / Accepted: May 23, 2021 / Published: May 23, 2021

This article is available from: https://e-epih.org/

(C) This is an open-access article distributed under the terms of the Creative Commons Attribution License (https://creativecommons.org/licenses/by/4.0/) which permits unrestricted use, distribution, and reproduction in any medium, provided the original work is properly cited.

(C) 2021, Korean Society of Epidemiology

\section{INTRODUCTION}

Systemic tumors, particularly cancers, are responsible for more than 9 million deaths worldwide every year. The most common organs affected are the breast, colon, and prostate. In 2018, there were 10,518 cancer deaths in Saudi Arabia. According to the Saudi tumor registry, the prevalence of brain tumors (mainly brain cancers) is relatively low compared with other regions around the world.

Primary central nervous system (CNS) tumors are a diverse group of tumors originating in the brain and spinal cord. More 
than $90 \%$ of these tumors arise in the brain and the remainder occur in the meninges, spinal cord, cranial nerves, and the surrounding structures. From the 1970s onwards, the diagnosis of CNS tumors has increased worldwide, with age-standardized incidence rates ranging from 4.3 to 18.6 per 100,000 per year. The highest incidence of CNS tumors is in northern Europe, followed by the United States, Canada, and Australia [1].

CNS tumors have been categorized into different histological groups by the World Health Organization (WHO) [2,3]. Unlike most other tumor types, CNS tumors are not staged; instead, they are classified as malignant or benign tumors based on a grading system, in which grade I/II tumors are considered "low grade-benign," whereas grade III/IV tumors are considered "high grademalignant." Given the differences in incidence rates across geographical locations and according to sex, there is a need to understand the etiology and risk factors of CNS tumors. The most common primary CNS tumor worldwide is glioma. The incidence of glioma is the highest in Australia, followed by Western Europe and North America [4]. The most common subtype is glioblastoma. The prevalence of glioblastoma in the United States in 2010 was estimated to be 47.6 per 100,000 [5]. In East Asia and Southern Europe, malignant meningioma is the most common malignant CNS tumor.

According to the Saudi cancer registry, the prevalence of brain cancers is relatively low in Saudi Arabia compared with other regions of the world. The most common malignant CNS tumor in Saudi Arabia is metastatic carcinoma followed by high-grade gliomas. Several studies found that the incidence of malignant CNS tumors is highest in males and in adults older than 40 years old [2], while benign tumors are more common in females [4]. In the pediatric population, the incidence of medulloblastoma is highest in Southern Europe and Eastern Europe, whereas astrocytic tumors are most common in the United States and Canada. There is no available data registry of CNS tumors in children in Saudi Arabia.

An expanded retrospective analysis of CNS tumors has never been conducted in Saudi Arabia. Only a single constrained retrospective analysis of cancer incidence was performed in the Eastern population of Saudi Arabia, and another general meta-analysis was performed that estimated brain cancer to account for $9.5 \%$ of all cancer diagnoses [6]. In the current study, we performed a retrospective analysis of CNS tumors in the Western Province of Saudi Arabia in the period 2014-2019. This study focused on generating a fine-grained epidemiological matrix, including age, sex, tumor location, histological subtype, genetic characteristics, and recurrence status. To our knowledge, this analysis is the first data registry of CNS tumors in Saudi Arabia that involves case distribution and an epidemiological matrix.

\section{MATERIALS AND METHODS}

\section{Study design and sample}

A cross-sectional study was performed using secondary data obtained from 3 out of 6 tertiary governmental hospitals affiliated with neuroscience centers, which receive cases of brain tumors: King Abdulaziz University (KAU) Hospital, King Abdulaziz Medical City in Jeddah (KAMC) and King Faisal Specialist Hospital and Research Centers in Jeddah (KFSHRC). Clinical data between 2014 and 2019, including age, sex, tumor location, histological classification, type of surgery, laboratory findings, and recurrence rates, were obtained. The dataset included 663 patients in adult and pediatric populations diagnosed with primary CNS tumors in the Western Province of Saudi Arabia.

\section{Methodology}

All cases were coded following the International Classification of Diseases for Oncology, third edition. The histological codes of tumors were then matched and grouped according to the categorization highlighted in the WHO 2016 classification of CNS tumors. Any tumors that lacked histological confirmation were removed from the dataset. Based on the diagnosed cases, the investigators identified primary CNS tumors under different categories. Patients under the age of 18 at the time of diagnosis were placed within the pediatric group. Although some cases had no genetic investigation, they were included in the analysis. IBM SPSS version 24 (IBM Corp., Armonk, NY, USA) and Microsoft Excel (Microsoft, Redmond, WA, USA) were used for the statistical analysis. A p-value $<0.01$ was considered to indicate statistical significance.

\section{Ethics statement}

The study was ethically approved by the National Biomedical Ethics Committee at KAU (HA-02-J-008) (Reference 190-19). This ethical approval was linked to additional approvals obtained from 2 medical centers (KAMC and KFSHRC). Following ethical approval, data on primary CNS tumors from the tumor registry were shared with the investigators. Ethics approval mandated that the researchers followed the procedures stipulated by "Good Clinical Practice" and the "Collaborative Institutional Training Initiative on Social and Behavioral Research."

\section{RESULTS}

Data on 663 patients with CNS tumors, diagnosed between 2014-2019, were retrieved from hospital records. As the tumor cases were collected over a 5-year period, some values of variables were inevitably missing. To overcome bias in the data analysis, these cases were excluded from the study, although cases with missing genetic results were included. The crude incidence rate was also difficult to calculate because there was no annual data registry from each hospital.

The cases enrolled in this study were from the local and expatriate populations. The cases included both adult and pediatric patients. Five hundred cases (75.4\%) were adults, and 163 cases (24.6\%) were children aged 18 or under, with a male-to-female ratio of 1.16. The mean age at diagnosis was $38.0 \pm 22.6$ years (Figure 1 and Table 1). Approximately $43.1 \%$ of the cases were in the 


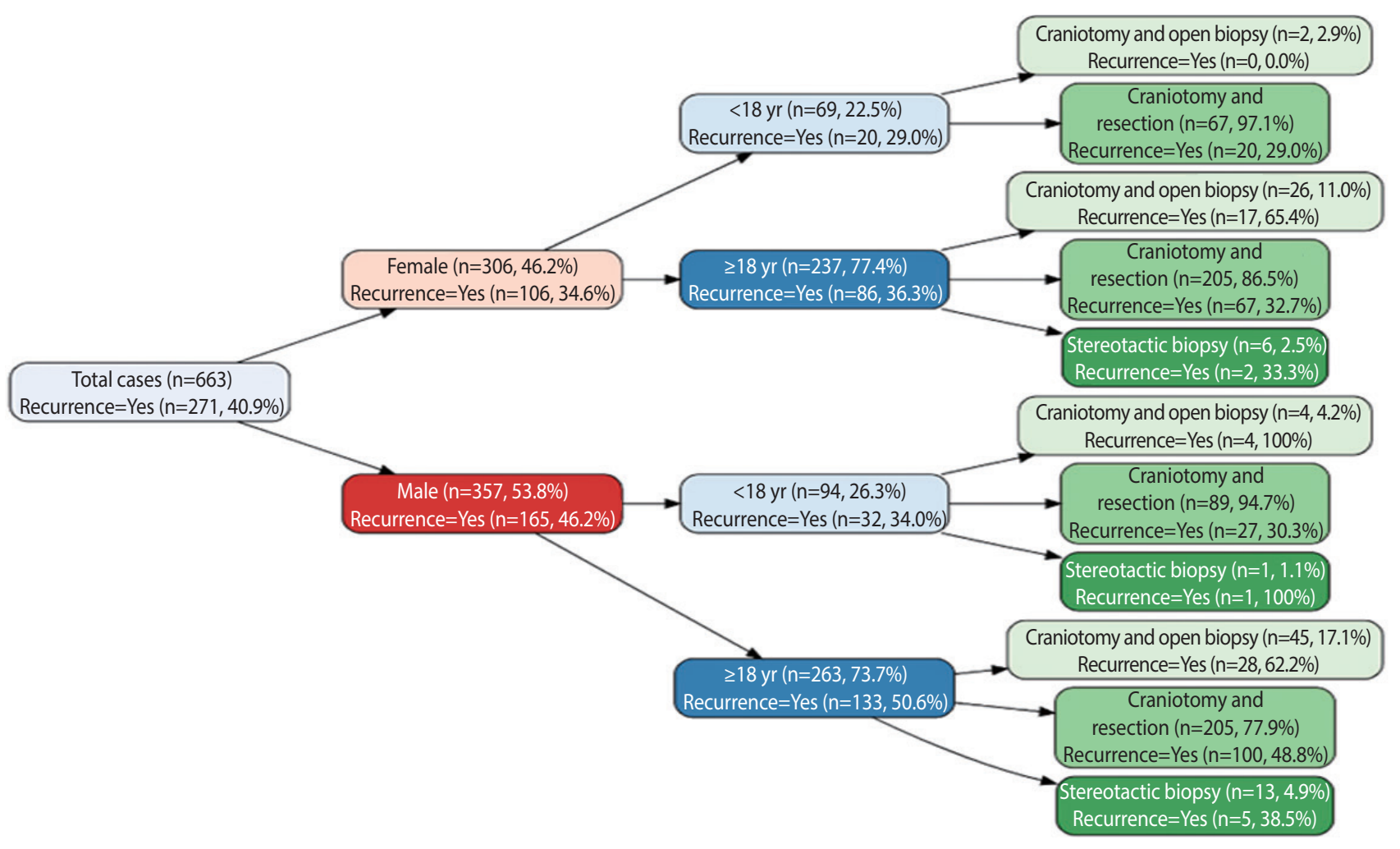

Figure 1. Distribution of central nervous system tumors by sex, age, type of surgical procedure, and recurrence rate.

25-54 years age group when diagnosed and $11.8 \%$ of the cases were diagnosed with a CNS tumor after the age of 65 years (Table 2).

The supratentorium was the most common location, with 515 cases $(77.7 \%)$, followed by 144 cases $(21.7 \%)$ in the infratentorium (posterior fossa, cerebellum, and fourth ventricle). The most predominant supratentorial site was the frontal lobe $(26.4 \%$, $\mathrm{n}=175$ ), in which intra-axial tumors (glioblastoma followed by oligodendroglioma) were the most commonly diagnosed tumors. Meningioma was the most common benign extra-axial tumor in the frontal lobe. Pilocytic astrocytoma $(n=70,10.6 \%)$ was the most common CNS tumors in the posterior fossa and predominantly occurred in pediatric patients. Overall, glioblastoma was the most common CNS tumor $(n=234,35.3 \%)$ followed by meningioma $(n=100,15.1 \%)$. Hence, low-grade tumors (WHO I/II) were more predominant in the CNS than high-grade tumors (WHO III/IV). Diffuse oligodendroglial tumors accounted for $5.0 \%$ of all glial tumors, whereas diffuse astrocytic tumors accounted for $2.6 \%$. Because glioblastoma with a primitive neuronal component (PNC) is rare and unique, it was categorized separately and accounted for $0 \%$ of all CNS tumors. Other tumors included dysembryoplastic neuroectodermal tumor, pineal gland tumors, subependymal giant cell astrocytoma, and choroid plexus papilloma, which together accounted for $3.9 \%$ of all CNS tumors (Figure 2).

The patients enrolled in this analysis commonly presented with headache (44.9\%, $\mathrm{n}=298)$. The next most common symptom was a focal neurological deficit $(19.9 \%, \mathrm{n}=132)$. Most adult cases who presented with seizures or epilepsy had glioblastoma or oligodendroglioma, while most pediatric cases who presented with seizure had either cortical pilocytic astrocytoma or ependymoma. Of the patients who presented with visual impairment, $5.0 \%$ of the total patients, enrolled in this study, were presented with visual impairment and their tumors were sellar, suprasellar, or optic meningiomas.

Approximately $85.4 \%(n=566)$ of the patients underwent surgical craniotomy and resection of the tumor, while 97 cases only underwent biopsy. The cases diagnosed via a brain biopsy were mostly high-grade gliomas and diffuse large B-cell lymphomas. The recurrence rate of CNS tumors was estimated to be approximately $41 \%$. The most recurrent tumors were classical glioblastoma $(63.5 \%, n=155)$, followed by glioblastoma with PNC and gliosarcoma. The recurrence rate was close to $0 \%$ in cases of hemangioblastoma, schwannoma, central neurocytoma, ganglioglioma and pilocytic/pilomyxoid astrocytoma. In the pediatric group, medulloblastoma showed low recurrence rate of $22.2 \%$. This was either because of the low volume of samples or because the patients received good management. Figure 3 summarizes the recurrence rate of the top $20 \mathrm{CNS}$ tumor types.

Our results also revealed that glioma represented the most common primary CNS tumor in the Western Province of Saudi Arabia, accounting for $62.3 \%$ of cases, with a male-to-female ratio of 1.5 (Table 3). Glioblastoma (WHO-IV glioma) was more prev- 
Table 1. Descriptive distribution of CNS tumors in the Western Province of Saudi Arabia $(\mathrm{n}=663)$

\begin{tabular}{|c|c|}
\hline Characteristics & n (\%) \\
\hline \multicolumn{2}{|l|}{ Sex } \\
\hline Female & $306(46.2)$ \\
\hline Male & $357(53.8)$ \\
\hline \multicolumn{2}{|l|}{ Age (yr) } \\
\hline$<18$ & $163(24.6)$ \\
\hline$\geq 18$ & $500(75.4)$ \\
\hline Mean $\pm S D$ [range] & $38.0 \pm 22.6[1.0-95.0]$ \\
\hline \multicolumn{2}{|l|}{ Clinical presentation } \\
\hline Cranial nerve palsy & $17(2.6)$ \\
\hline Focal neurological deficit & $132(19.9)$ \\
\hline Headache & $298(44.9)$ \\
\hline Other & $60(9.0)$ \\
\hline Seizure & $123(18.6)$ \\
\hline Visual impairment & $33(5.0)$ \\
\hline \multicolumn{2}{|l|}{ Type of surgery } \\
\hline Craniotomy and open biopsy & $77(11.6)$ \\
\hline Craniotomy and tumor resection & $566(85.4)$ \\
\hline Stereotactic biopsy & $20(3.0)$ \\
\hline \multicolumn{2}{|l|}{ Tumor location } \\
\hline Third ventricle & $2(0.3)$ \\
\hline Fourth ventricle & $4(0.6)$ \\
\hline Basal ganglia & $4(0.6)$ \\
\hline Brainstem & $8(1.2)$ \\
\hline Cerebellum & $15(2.3)$ \\
\hline Cerebellopontine angle & $18(2.7)$ \\
\hline Intradural extramedullary & $2(0.3)$ \\
\hline Frontal & $175(26.4)$ \\
\hline Interhemispheric & $3(0.5)$ \\
\hline Intramedullary & $2(0.3)$ \\
\hline Jugular foramen & $1(0.2)$ \\
\hline Lateral ventricle & $32(4.8)$ \\
\hline Occipital & $22(3.3)$ \\
\hline Olfactory groove & $5(0.8)$ \\
\hline Optic chiasm & $3(0.5)$ \\
\hline Optic nerve & $5(0.8)$ \\
\hline Parasagittal & $11(1.7)$ \\
\hline Parietal & $97(14.6)$ \\
\hline Pineal region & $4(0.6)$ \\
\hline Planum & $4(0.6)$ \\
\hline Posterior fossa & $98(14.8)$ \\
\hline Sellar & $1(0.2)$ \\
\hline Sphenoid wing & $12(1.8)$ \\
\hline Suprasellar & $21(3.2)$ \\
\hline Temporal & 99 (14.9) \\
\hline Thalamic & $15(2.3)$ \\
\hline \multicolumn{2}{|l|}{ Recurrence status } \\
\hline No recurrence & $392(59.1)$ \\
\hline Recurrence & $271(40.9)$ \\
\hline
\end{tabular}

CNS, central nervous system; SD, standard deviation.
Table 2. Age distribution of CNS tumor patients in the Western Province of Saudi Arabia

\begin{tabular}{lc}
\hline Age at diagnosis & $\mathrm{n}(\%)$ \\
\hline$<14$ & $144(21.7)$ \\
$15-24$ & $52(7.8)$ \\
$25-54$ & $286(43.1)$ \\
$55-64$ & $103(15.5)$ \\
$\geq 65$ & $78(11.8)$ \\
Total & $663(100)$ \\
\hline
\end{tabular}

CNS, central nervous system.

alent in male patients $(64.3 \%, \mathrm{n}=157)$ than in female patients ( $35.7 \% . n=87$ ). Oligodendroglioma was the only grade II glioma that was slightly more prevalent in females than males, with a difference of only 2 cases. There was a significant difference in the age distribution between adult and pediatric gliomas $(\mathrm{p}<0.01)$. CNS gliomas were more prevalent in adult patients $(79.2 \%, \mathrm{n}=327)$ than in pediatric patients $(20.8 \%, \mathrm{n}=86)$. Glioblastoma was the predominant CNS glioma in adult patients, 95.5\% (233/344) of the glioblastoma found in adult. The glioma that showed the strongest predilection for pediatric patients was pilocytic astrocytoma $(83.3 \% . n=60)$, which was mainly found in the posterior fossa. Approximately $8 \%$ of oligodendroglioma cases were children. There was a significant difference in tumor location among all CNS gliomas $(\mathrm{p}<0.001)$. The frontoparietal area was the most predominant location for glioblastoma (70-87\%). Oligodendroglioma was predominantly observed in the frontal lobe $(21 / 34$ cases; $61.8 \%$ ).

The most common clinical features associated with CNS gliomas are headache and focal neurological deficit, which commonly accompany glioblastoma and cortical pilocytic astrocytoma. Epilepsy was frequently associated with glioblastoma $(40.1 \%, n=97)$ and oligodendroglioma $(58.3 \%, \mathrm{n}=21)$. Our results also showed that most high-grade gliomas were enhanced on magnetic resonance imaging (MRI) after gadolinium contrast. Oligodendrogliomas and pilocytic astrocytomas also showed partial enhancement on MRI.

The methylation status of methylguanine methyl transferase (MGMT) was not routinely assessed in all CNS gliomas. In the current data, we found that MGMT was methylated in 26/31 cases of glioblastoma that were tested. Other CNS gliomas, such as pilocytic astrocytoma or oligodendroglioma, did not show MGMT methylation. Because isocitrate dehydrogenase 1 (IDH1) testing is not routinely performed in most medical centers, few tumors were tested for IDH1 mutation via immunohistochemistry or gene sequencing. Therefore, out of the 104 glioblastoma cases, 62 (59.0\%) were wild-type for IDH1 and $42(40.2 \%)$ carried an IDH1 mutation.

Most of the CNS gliomas (81.6\%) underwent complete surgical resection. Non-resection of tumors was because of surgical inaccessibility or poor patient health status. In 10 cases of non-resected glioblastoma, the tumor was located in a subcortical structure (basal ganglia or thalamus). There was a significant differ- 


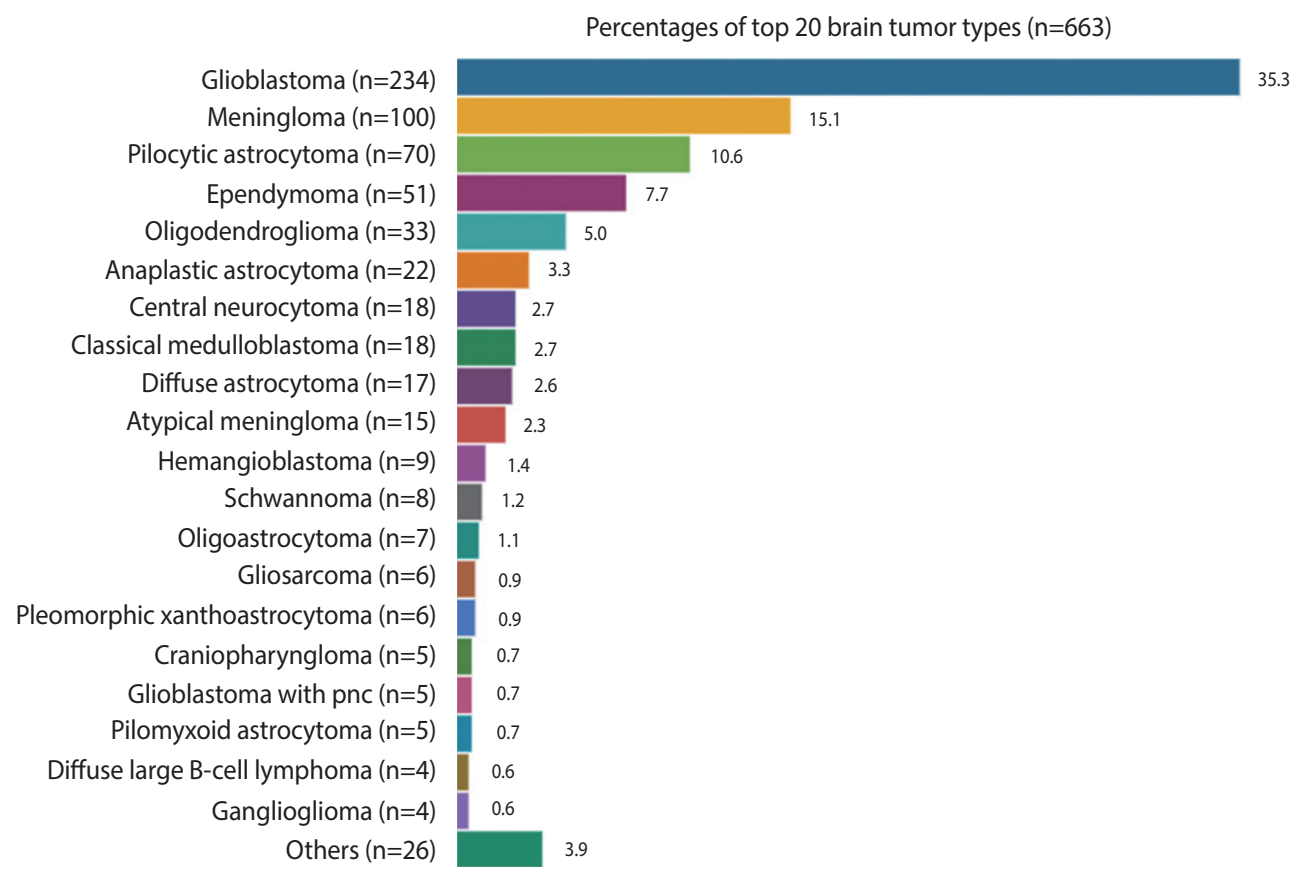

Figure 2. The incidence of central nervous system tumors in the Western Province of Saudi Arabia in the period $2014-2019$.

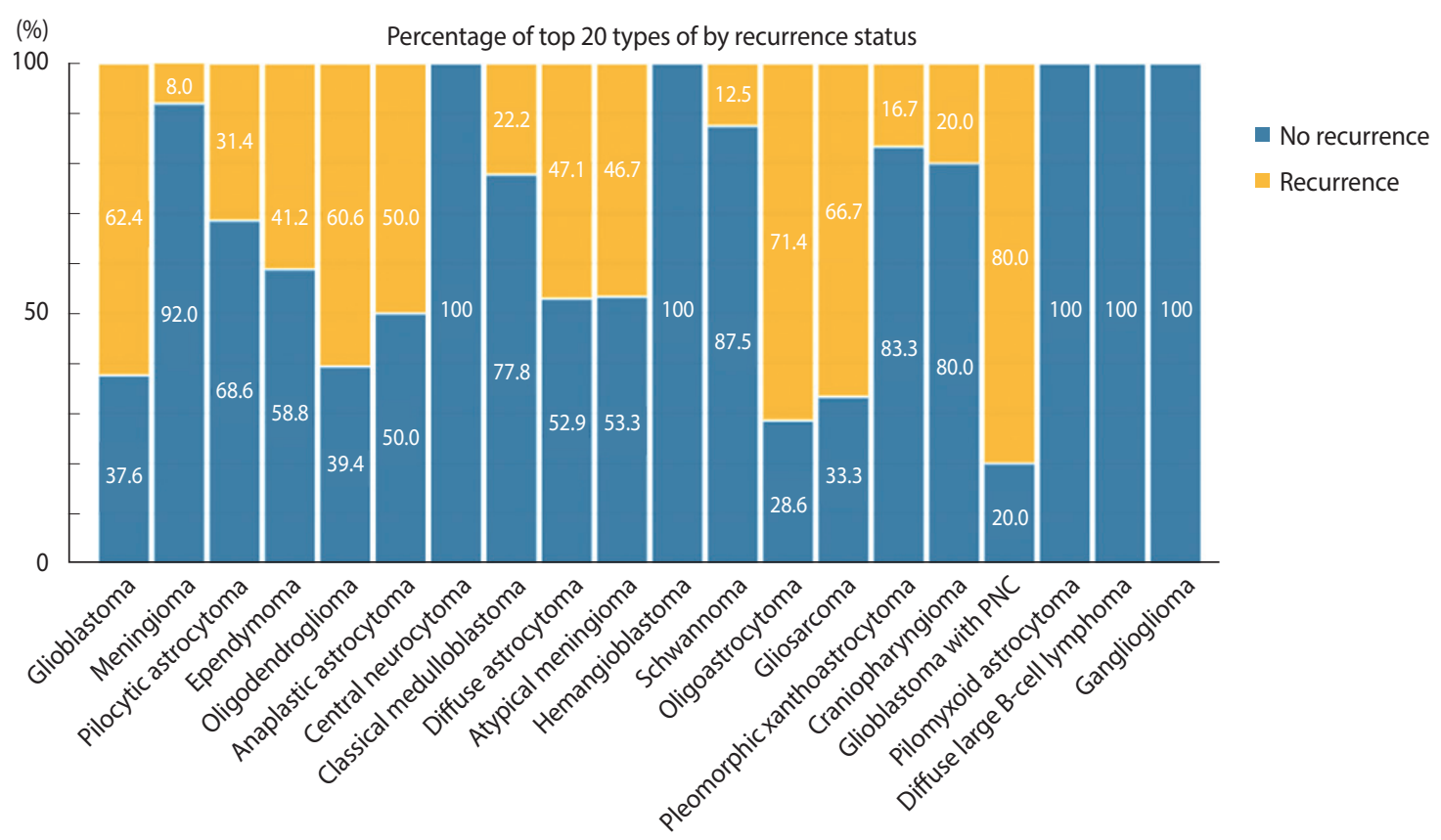

Figure 3. The recurrence status of the most common central nervous system tumors in the Western Province of Saudi Arabia.

ence in recurrence rate among CNS gliomas $(\mathrm{p}<0.001)$. The recurrence rate was the highest among glioblastoma patients $(63.5 \%)$ and the lowest was among pilocytic astrocytomas (69.4\%) (Figure 4).

\section{DISCUSSION}

The highest incidence of CNS tumors is in Northern Europe, followed by the United States, Canada, and Australia [1]. Given the difference in incidence rates across geographical locations and according to sex, there is a need to understand the etiology and 


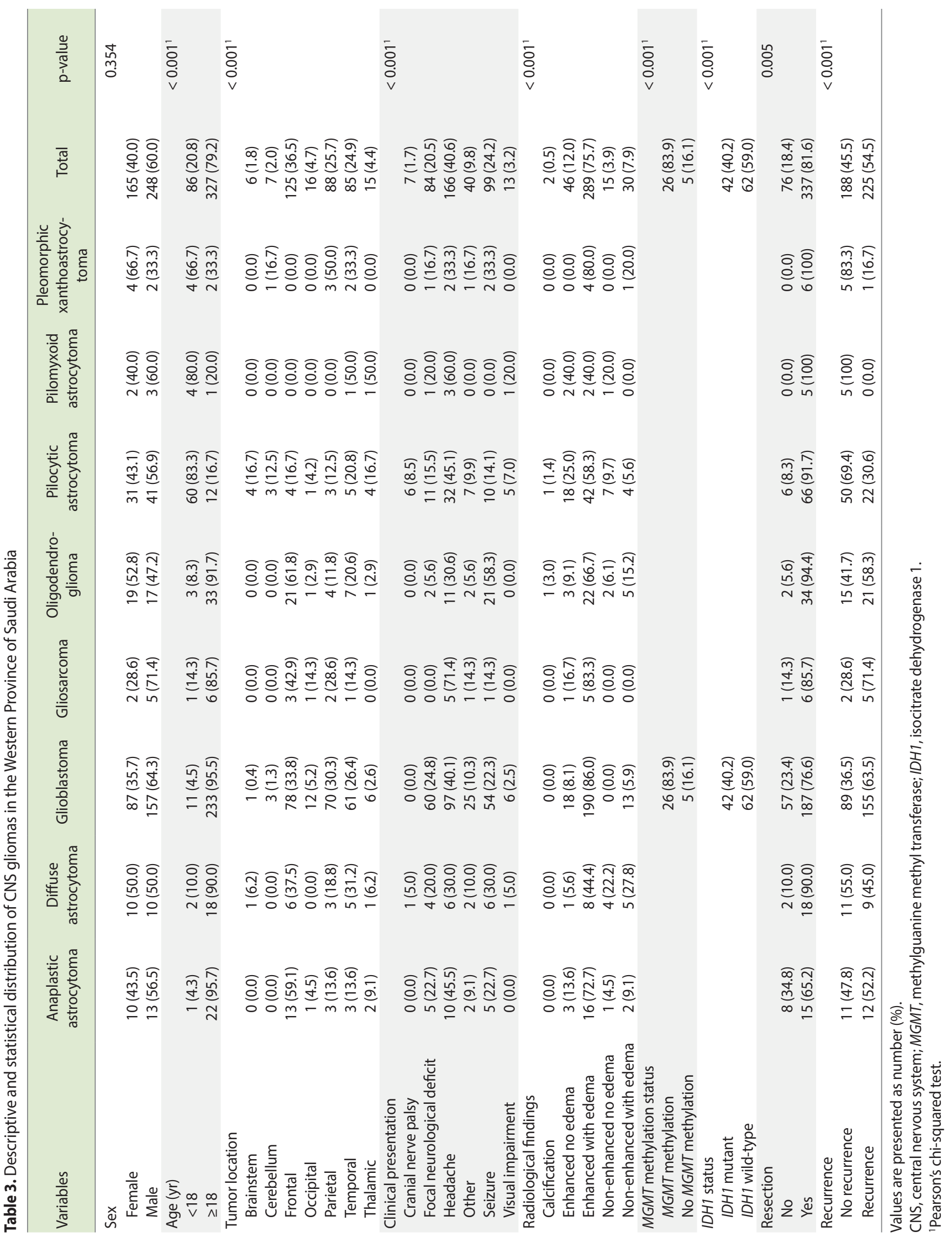




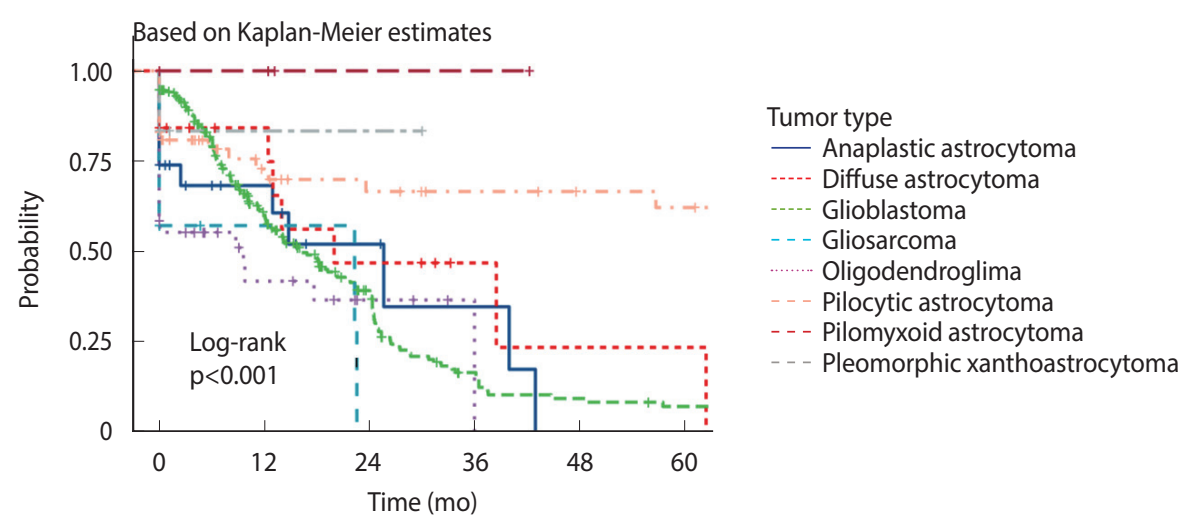

Figure 4. Recurrence interval for common central nervous system gliomas in the Western Province of Saudi Arabia.

risk factors of CNS tumors [2,3]. The most common CNS tumors were grade VI, which was reinforced by the high number of gliomas recorded in the limited registry. However, the second most common tumor was benign meningioma (WHO grade I). With health insurance and government-funded hospital treatment, the likelihood of cases going undiagnosed has decreased.

Barnholtz-Sloan et al. [4] found that the global incidence of malignant CNS tumors was highest in male patients and adults older than 40 , while benign tumors were found to be more common in female patients. Among children less than 15 years old, the highest incidence of CNS tumors was in children aged between 0-4 years. In the Western Province of Saudi Arabia, the mean age at diagnosis of all CNS tumors in males and females was 38 years. This is different from the United States, where the mean age is 59 years, but is similar to the United Arab Emirates, where the mean age is 33.4 years [7]. The predominant age at CNS tumor diagnosis was between 25-54 years. There was no significant difference in the sex distribution of all CNS tumors in the Western Province of Saudi Arabia (male-to-female ratio: 1.16). The highest incidence of CNS tumors was in children aged between 0 - 14 years.

The incidence of gliomas is highest in Australia, followed by Western Europe and North America [4]. The most common subtype is glioblastoma. Although most CNS tumors diagnosed in the United States are benign, the prevalence of glioblastomas in 2010 was estimated to be 47.6 per 100,000 (103,634 total cases) [5]. In the period from 2014 to 2019 in the Western Province of Saudi Arabia, the most commonly diagnosed CNS tumors were also gliomas, mainly glioblastoma (35.3\%). Most of these glioblastomas were supratentorial (frontoparietal area), IDH1 wild-type, MGMT non-methylated, and associated with poor outcomes and a high recurrence rate $[8,9]$. Most common pediatric tumors diagnosed in the Western Province of Saudi Arabia were pilocytic astrocytomas that were predominantly found in the posterior fossa. In contrast, diffuse astrocytoma and embryonic tumors are commonly diagnosed in pediatric patients in the United Arab Emirates [7].

After diagnosis with a malignant CNS tumor, the 1-year relative survival rate was $56.6 \%$, with a 5 -year relative survival rate of
$32.1 \%$ [4]. Mortality caused by malignant CNS tumors also varies across the globe [1]. The mortality is highest in Northern Europe. In the Western Province of Saudi Arabia, most CNS gliomas (81.6\%) underwent complete surgical resection. Non-resection of tumors was because of surgical inaccessibility or poor patient health status. There was also a significant difference in the recurrence rate among CNS gliomas $(\mathrm{p}<0.001)$. The recurrence rate was highest among glioblastoma patients and lowest among those with pilocytic astrocytoma (Figure 4).

The only limitation acknowledged in this study is a lack of other specific epidemiological parameters (such as mortality rate) and non-registered cases from non-tertiary hospitals. However, this study is novel in that it presents the corresponding data from this region for the first time, which included age, sex, location and histological types, as well as the distribution of brain tumors in the Western Province of Saudi Arabia, which is notable for having higher levels of immigrants and expatriates than other local regions.

In conclusion, the incidence, prevalence, management, and recurrence of CNS tumors vary according to histological type, age at diagnosis, sex, and available treatments. A data registry of CNS tumors for each hospital is encouraged. Further research should be performed to identify potential risk factors for CNS tumors in Saudi Arabia. Genome technology (including genomic, epigenomic, transcriptomic, proteomic, and metabolomic approaches) is important in the healthcare system and provides an opportunity to identify relationships between the incidence rate and risk factors. Further large, multicenter epidemiological studies as well as well-annotated omics datasets are important for advancing CNS tumor research.

\section{CONFLICT OF INTEREST}

The authors have no conflicts of interest to declare for this study.

\section{FUNDING}

None. 


\section{ACKNOWLEDGEMENTS}

None.

\section{AUTHOR CONTRIBUTIONS}

Conceptualization: MK. Data curation: SB, YM, AB, RS, AIL. Formal analysis: NSB. Funding acquisition: None. Project administration: MK. Visualization: BA. Writing - original draft: $\mathrm{MK}$, BA, NSB, SB, YM, AB, RS, AIL. Writing - review \& editing: MK.

\section{ORCID}

Maher Kurdi: https://orcid.org/0000-0002-8979-3849; Nadeem Shafique Butt: https://orcid.org/0000-0002-0473-4920; Saleh Baeesa: https://orcid.org/0000-0002-3053-7912; Badrah Alghamdi: https://orcid.org/0000-0002-9411-3609; Yazid Maghrabi: https://orcid. org/0000-0003-0749-7307; Anas Bardeesi: https://orcid.org/00000003-3154-8688; Rothaina Saeedi: https://orcid.org/0000-00019358-6925; Ahmed I. Lary: https://orcid.org/0000-0001-7137-9992

\section{REFERENCES}

1. Ferlay J, Soerjomataram I, Dikshit R, Eser S, Mathers C, Rebelo M, et al. Cancer incidence and mortality worldwide: sources, methods and major patterns in GLOBOCAN 2012. Int J Cancer 2015; 136:E359-E386.

2. Ostrom QT, Gittleman H, Liao P, Vecchione-Koval T, Wolinsky Y,
Kruchko C, et al. CBTRUS statistical report: primary brain and other central nervous system tumors diagnosed in the United States in 2010-2014. Neuro Oncol 2017;19(suppl 5):v1-v88.

3. Louis DN, Perry A, Reifenberger G, von Deimling A, FigarellaBranger D, Cavenee WK, et al. The 2016 World Health Organization classification of tumors of the central nervous system: a summary. Acta Neuropathol 2016;131:803-820.

4. Barnholtz-Sloan JS, Ostrom QT, Cote D. Epidemiology of brain tumors. Neurol Clin 2018;36:395-419.

5. Zhang AS, Ostrom QT, Kruchko C, Rogers L, Peereboom DM, Barnholtz-Sloan JS. Complete prevalence of malignant primary brain tumors registry data in the United States compared with other common cancers, 2010. Neuro Oncol 2017;19:726-735.

6. Alqahtani WS, Almufareh NA, Domiaty DM, Albasher G, Alduwish MA, Alkhalaf H, et al. Epidemiology of cancer in Saudi Arabia thru 2010-2019: a systematic review with constrained meta-analysis. AIMS Public Health 2020;7:679-696.

7. Khan S, Kambris ME, AlShamsi ET. Epidemiology of brain tumors in the United Arab Emirates: a national registry cross-sectional study. BMC Neurol 2020;20:301.

8. Capper D, Weissert S, Balss J, Habel A, Meyer J, Jäger D, et al. Characterization of $\mathrm{R} 132 \mathrm{H}$ mutation-specific IDH1 antibody binding in brain tumors. Brain Pathol 2010;20:245-254.

9. Wiestler B, Capper D, Holland-Letz T, Korshunov A, von Deimling A, Pfister SM, et al. ATRX loss refines the classification of anaplastic gliomas and identifies a subgroup of IDH mutant astrocytic tumors with better prognosis. Acta Neuropathol 2013; 126:443-451. 